\title{
Refining structures against reflection rank: An alternative metric for electron crystallography.
}

\author{
Alexander S. Eggeman and Paul A. Midgley * \\ Department of Materials Science and Metallurgy, University of Cambridge, \\ Pembroke Street, Cambridge, CB2 3QZ U.K.E-mail: ase25@cam.ac.uk, \\ pam33@cam.ac.uk
} (Received 0 XXXXXXX 0000; accepted 0 XXXXXXX 0000)

\begin{abstract}
A new metric is proposed to improve the fidelity of structures refined against precession electron diffraction data. The inherent dynamical nature of electron diffraction ensures that direct refinement of recorded intensities against structure factor amplitudes can be prone to systematic errors. Here we show that the relative intensity of precessed reflections, their rank, can be used as an alternative metric for refinement. Experimental data from erbium pyrogermanate shows that applying precession reduces the dynamical transfer of intensity between reflections and hence stabilises their rank, enabling accurate and reliable structural refinements. This approach is then applied successfully to an unknown structure of an oxygen-deficient bismuth manganite resulting in a refined structural model that is similar to a calcium analogue.
\end{abstract}

\section{Introduction}

A primary goal of crystallography is to accurately determine the arrangement of atoms within a crystal structure. There are techniques that allow this to be performed 
directly, such as high resolution TEM (Hovmöller et. al. 2002) and STEM (Pyrz et. al. 2008) both of which have benefitted in recent years from the development of lens correctors to minimise optical aberrations (Haider et. al. 1998, Krivanek et. al. 1999). However diffraction-based techniques remain a popular tool for electron crystallographers because of the robust procedures developed to record experimental data, the absence of imaging lenses (meaning optical deficiencies do not limit the resolution of the data) and the availability of powerful computer algorithms to transform between reciprocal and real space densities, allowing structure solution and refinement to be performed at high-speeds (Saxton et. al. 1979, Frigo \& Johnson 2005, Burle et. al. 2007).

A major obstacle for electron crystallographers arises from the strong interaction between the electron beam and the crystal potential of the lattice that leads to multiple scattering and dynamical diffraction. Multiple scattering ensures that, in general, each diffracted intensity, $I_{g}$, cannot be related simply to its corresponding structure factor, $F_{g}$ but is instead a complex function of all the structure factors, making direct use of diffraction intensities for structure solution difficult, if not impossible. This is true also for structure refinement, in which a trial structure is altered in order to best fit a set of experimental data. For X-rays, for example, a kinematical refinement is normally used where each intensity is compared to the square of the corresponding structure factor for a trial structure. However, for electrons this is not always applicable and a full dynamical refinement is then needed (Jansen et. al. 1998).

To improve the applicability of electron data to structure solution, one technique that has become increasingly popular is precession electron diffraction, or PED (Vincent \& Midgley 1994). By precessing the incoming beam about a zone axis, the effects of multi-beam dynamical scattering are reduced overall. Bringing the beam back onto the optic axis below the specimen allows the geometry and symmetry of the zone- 
axis pattern to be maintained and PED intensities are integrated through the Bragg condition. In many circumstances, PED intensities can be then treated as kinematical at least in terms of their use in structure solution algorithms (Ciston et. al. 2008, White et. al. 2010, Eggeman et. al. 2010, Klein \& David 2010), but this does not always extend to structure refinement, which uses all reflections, often weighted equally, rather than the subset of strong reflections that tend to dominate structure solution techniques.

In order to use PED data for refinement we consider features of the diffraction data which can be used for an alternative metric. From previous studies (Eggeman et. al. 2010, Barnard et. al. 2010) the precession geometry (with sufficiently high precession angle) has been shown to minimise the multi-beam effects involving the low order reflections in the pattern. Since these low order reflections tend to be among the strongest in the pattern, any remaining multi-beam dynamical effects will tend to involve weaker reflections, and result in smaller transfers of intensity. So, while the absolute value of intensity can still be perturbed by dynamical effects, the overall rank (most intense to least intense) of the reflections becomes considerably more stable when precession is applied. In this article we describe the use of reflection rank as a metric for structure refinement from PED data.

\section{Rank-Correlation Analysis}

The typical method to determine the agreement between a set of observed (experimental) diffraction intensities $\left(I_{o b s}\right)$ and those intensities calculated from a structural model $\left(I_{\text {calc }}\right)$ is to use the sum of residual differences $(R)$ between the two sets of reflections:

$$
R=\frac{\sum_{h}\left|I_{o b s}(h)-K I_{\text {calc }}(h)\right|}{\sum_{h}\left|I_{o b s}(h)\right|}
$$

IUCr macros version 2.1.4: 2010/12/07 
where $\mathrm{K}$ is a scaling factor such that:

$$
K=\frac{\sum_{h}\left|I_{\text {obs }}(h)\right|}{\sum_{h}\left|I_{\text {calc }}(h)\right|}
$$

The new approach taken in this work is to use a secondary quantity of the reflection intensities, their relative intensity or rank. In this case a set of $n$ observed intensities of reflections $\left(I_{1,1}, I_{1,2}, \ldots I_{1, n}\right)$, were ordered from most intense to least intense, the most intense reflection was assigned a rank of 1 through to the least intense being assigned a rank of $n$ leading to $\left(r_{1,1}, r_{1,2}, \ldots r_{1, n}\right)$. An identical process was performed on the calculated reflection set to produce a second set of reflection ranks $\left(r_{2,1}, r_{2,2}, \ldots r_{2, n}\right)$. The correlation factor $(C)$ of these two sets of ranks was calculated using:

$$
C=\frac{n \sum_{h=1}^{n} r_{1, h} \cdot r_{2, h}-\sum_{h=1}^{n} r_{1, h} \cdot \sum_{h=1}^{n} r_{2, h}}{\left(\left(n \sum_{h=1}^{n} r_{1, h}^{2}-\sum_{h=1}^{n} r_{1, h} \cdot \sum_{h=1}^{n} r_{1, h}\right) \cdot\left(n \sum_{h=1}^{n} r_{2, h}^{2}-\sum_{h=1}^{n} r_{2, h} \cdot \sum_{h=1}^{n} r_{2, h}\right)\right)^{1 / 2}}
$$

where $h$ is the same reflection index in both sets. Unlike the $R$-factor calculated in Eq.1, which becomes smaller as the agreement between the two reflection sets improves, the rank correlation factor (Eq. 3) will increase as the agreement between the two reflection sets improves to a maximum value of 1 (indicating perfect correlation or identical reflection ranks in both sets). This metric was incorporated into a LevenbergMarquadt refinement algorithm (Levenburg 1944, Marquadt 1962), which was written to maximise the rank correlation.

\section{Results}

All PED experiments were performed on a Philips CM30 TEM using a Nanomegas SpinningStar precession apparatus. Diffraction patterns were recorded on Ditabis imaging plates.

IUCr macros version 2.1.4: 2010/12/07 


\subsection{Erbium Pyrogermanate - a test case}

Erbium pyrogemanate $\left(\mathrm{Er}_{2} \mathrm{Ge}_{2} \mathrm{O}_{7}\right.$ - EGO) was used as a test structure. Whilst this oxide has been studied extensively (Smolin 1970, Midgley \& Saunders 1996, Sleight 1997, Eggeman et. al. 2010) it remains a useful test material because it contains a combination of heavy, medium and light atoms and has a sufficiently complex structure to give a wide range of diffraction intensities and a highly non-monotonic variation of intensity with increasing scattering angle. The material occupies the tetragonal space group $P 4_{1} 2_{1} 2$ with cell parameters $\mathrm{a}=6.778 \AA$ and $\mathrm{c}=12.34 \AA$. As a proof of concept, kinematically ideal reflection intensities (with $s \leq 0.41 \AA^{-1}$ ) for the [001] zoneaxis diffraction pattern were calculated from the accepted atomic co-ordinates for EGO then structure solutions were recovered using the tangent formula from these intensities. The heavy atom (Er and Ge) positions were determined from this map and a Fourier difference synthesis was used to identify the oxygen positions. This recovered model was refined using the rank-refinement technique and was able to return a structure with a rank-correlation of 0.99 , the refined atomic co-ordinates in this $x-y$ projection (shown in Table 1 ) agree extremely well with the ideal values.

A series of PED patterns were recorded parallel to the [001] zone-axis (projected plane group symmetry $p 4 g m$ ) of an EGO crystal with precession angles ranging between 0 and 50 mrad, examples of which are shown in Figure 1. The crystal thickness was determined to be $52 \mathrm{~nm}$ after a best-fit match of unprecessed diffraction intensities with multislice simulations of the accepted structure. One limitation of using PED on crystals with a primitive Bravais lattice is that at high precession angles, HOLZ reflections can overlap directly with ZOLZ reflections and so to avoid this 'HOLZ-creep' the data was restricted to a subset of ZOLZ reflections with $s \leq 0.85 \AA^{-1}$ giving a final total of 36 independent reflections. The intensity of the reflections were calculated as the average between symmetrically equivalent reflections $\left(R_{\text {sym }}\right.$ lay between 0.008 and IUCr macros version 2.1.4: 2010/12/07 
0.017), the only exception was for the $0 h 0=2 n+1$ which were excluded from the refinement as they are forbidden by the space group. From the experimental ZOLZ data, structure solutions were recovered using a Tangent Formula implementation (Giacovazzo 1998). Structural models of the heavy atoms (erbium and germanium) in these solutions were extracted and used as initial structures in the rank-correlation refinement procedure described in the previous section. Oxygen positions could not be satisfactorily determined from the direct methods solutions and so were not included in the refinement.

Figure 2 shows two scatter plots comparing the ranks of reflections in the $40 \mathrm{mrad}$ precession angle experimental data-set on the ordinate with the rank of the corresponding reflection generated from the structural model on the abscissa. Figure 2a uses the reflection rank of the initial structural model reflections while Figure $2 \mathrm{~b}$ shows those for the refined structural model. The improvement in the correlation (approach to a straight line) for the refined data is evident with only a few reflections lying outside the main trend after refinement. The initial and final 'rank-correlation' factor for all data sets in the precession series are shown in Figure 3. From this graph it can be seen that the refinement was able to significantly increase the rank-correlation for all sets of diffraction data recorded at different precession angles. Increasing the precession angle tends to produce better quality starting structure models and also leads to refined solutions with higher rank-correlation factors. The question remains as to whether these rank-refined solutions are genuinely accurate structural estimates or are simply local minima in the allowed structure-space.

Two comparisons were performed to verify the suitability of the final structures. Firstly, the conventional residual $R$-factors were calculated between the experimental intensities and intensity values generated from the initial and final structure models (Figure 4a). The reduction in this residual shows that the improvement in rank- 
correlation is associated with a better agreement between the model intensities and the experimental diffraction data. For a further comparison, an $R$-factor calculation was performed between kinematical ideal intensities and intensities calculated from the final structural model, this is shown as the final (kinematical) data. The residual values between these reflection sets are improved compared to values calculated from the experimental intensities at moderate to high precession angles. This suggests that the rank-correlation refinement is able to produce a model closer to the ideal than is possible using refinement of the electron data based on the (conventional) $R$-factor metric. The final set of values were refinements performed using a conventional $R$ factor calculation. These confirm that using the regular approach to refinement for this particular set of diffraction data would lead to structures that are much less reliable than those returned by the rank-correlation refinement procedure.

Secondly, the atomic co-ordinates of the heavy atoms columns in the refined structure were compared with the accepted structural model (Figure 4b). Since the calculated $R$-factors are in the acceptable range $(R \leq 0.3)$ only for precession angles of 30mrad and above, this comparison is shown only for this range where reasonable structural models have been returned. Both the erbium and germanium atoms occupy $8 b$ Wyckoff sites in the $P 4_{1} 2_{1} 2$ structure so only 4 independent structural parameters (Er- $x$, Er- $y$, Ge- $x$ and Ge- $y)$ are required. This data shows that for the range of precession angles where acceptable structural models have been returned, the refinement leads to a significant reduction in the displacement of the atomic columns from their ideal positions.

\subsection{Oxygen Deficient Bismuth Manganite}

The results for erbium pyrogermanate suggest that the rank of reflections can be a powerful metric for refining structural models using PED data. As a second test,

IUCr macros version 2.1.4: 2010/12/07 
a sample of a newly discovered oxygen-deficient bismuth manganite was investigated. This material has been reported (Eggeman et. al. 2011) to crystallise into an $n=2$ Ruddlesden-Popper phase (shown schematically in Figure 5a) comprising 2 layers of a perovskite sub-structure interspersed with a layer of a rock-salt structure. PED data was recorded parallel to the [001] zone-axis of the crystal using a precession angle of 30mrad (Figure 5b). By determining the whole-pattern symmetry of this axial projection along with other major zone-axis patterns using HOLZ reflections and CBED analysis where necessary, it was determined that the structure occupies the orthorhombic non-centrosymmetric $C m c 2_{1}$ space-group, with cell parameters $\mathrm{a}=16.8 \AA, \mathrm{b}=5.51 \AA$ and $\mathrm{c}=5.45 \AA$.

Refinement of the structure was performed using the rank-correlation as the metric as described before with a total of 14 independent atomic coordinate parameters ( $x$ and $y$ for 7 independent atomic sites) refined against 145 independent reflections $\left(R_{\text {sym }}=\right.$ 0.032). The process was performed in two stages. Firstly, the independent heavy atom columns (two bismuth and one manganese) were refined from positions set by an ideal perovskite/rock-salt cell. Then the coordinated oxygen octahedra were added around the manganese sub-lattice positions and the combined structure refined for a second time. The model was used for the initial atomic positions because estimates of the oxygen positions were impossible to determine from structure solutions recovered from the experimental diffraction data. The result from maximising the rank-correlation is shown in Figure 6, which comprises two scatter plots, plotted in the same way as Figure 2. Figure 6a shows the rank of the reflections generated from the initial structural model while Figure $6 \mathrm{~b}$ shows the ranks from the final refined model. The overall reduction in the spread of points towards the ideal straight line is clear and, importantly, there is a region over $c a$. the first 50 reflections (the strongest) where the correlation is even better. This all suggests that while the refinement may not 
have exactly matched the measured intensities, there is a good agreement in the order of the most significant portion of the diffraction pattern, resulting in this case, in an improvement in the rank-correlation factor from 0.44 to 0.79 . There are apparent exceptions from this general improvement, notably a small cluster of points at the bottom right of Figure 6b. These few points represent several reflections with some of the smallest Bragg angles in the pattern. Here, the overlap between the direct beam and these reflections caused a very high background to the reflections and likely significant under-estimation of their true intensity and consequently an incorrect rank.

The structural model produced by this refinement is shown in Figure 7a. Whilst there is no previously reported structure for this particular material, there is however a calcium analogue structure (Elcombe et. al. 1991) and the same projection of this analogue structure is shown for comparison in Figure $7 \mathrm{~b}$. There are notable similarities in the relative positions of the different atomic columns within this structure. The major differences in the structure seem to be that the bismuth-oxygen columns (indicated in Figure 7a) have a more significant 'zig-zag' displacement compared to the corresponding calcium-oxygen columns. This behaviour is not unexpected as the bismuth ion carries a lone-pair of $6 s$ electrons in its outer shell, which we might expect to cause it to reside further from the centre of the perovskite cell. The other significant difference is that one of the oxygen columns has separated into a doublet in projection in the calcium manganite structure (indicated in Figure 7b) but remains essentially a singlet in the bismuth manganite. In the original reported structure (Elcombe et.al. 1991) this column was adjusted to match the doublet offset in the other columns (in our study the offset was not applied post-refinement). The comparison of the refined structure and the calcium analogue structure are shown in Table 2.

IUCr macros version 2.1.4: 2010/12/07 


\section{Discussion}

The use of rank as a metric for the refinement of electron diffraction data is driven by one important consideration, that electron diffraction data is always affected by dynamical scattering. Applying a sufficiently high precession angle to the beam can help to avoid $n$-beam multiple scattering involving low-order reflections and since these are often the strongest reflections they contribute substantially more to dynamical scattering. However there will always be some transfer of intensity between reflections within an electron diffraction pattern. As such the use of kinematical intensities calculated directly from structure factors can never be a wholly accurate way of representing the scattering from the crystal structure under investigation. The rank of a reflection offers an alternative means of representing the scattering from the crystals, one in which small dynamical perturbations can be accommodated.

This can be seen in the structures refined from PED data from erbium pyrogermanate. The comparison of the intensity residual from the experimental data and that from the kinematical data (Fig. 3b) shows that for small precession angles, persistent dynamical effects alter the intensities sufficiently to make it difficult to solve the structure satisfactorily. These same effects mean that the rank of the reflections is unsuitable to refine the structure accurately. However at moderate to high precession angles the reduction in strong dynamical effects is sufficient to stabilise the relative intensities (and hence the ranks) of the majority of reflections while there are still variations in the corresponding absolute intensity values. The resulting rankcorrelation refinement can therefore allow better quality structures to be found than the corresponding conventional $R$-factor for PED data.

The results from the bismuth manganite sample show that for a partially complete structure, rank-correlation refinement can return structures that are improved. The presence of strong scatterers, such as bismuth, in this structure means that dynamical 
effects are likely to be significant. It is therefore extremely unlikely that a pseudokinematical condition will be easily reached for this structure and so the use of a novel refinement metric is required.

\section{Conclusions}

Two case studies have been shown to highlight the potential use of a rank-correlation factor as an improved metric for the refinement of structures using precession electron diffraction data. The heavy atom positions in erbium pyrogermanate have been refined to high accuracy and, importantly, there is clear evidence that the rank-correlation factor is less sensitive, compared to the conventional $R$-factor, to dynamical perturbations that exist even at large precession angles. The technique was applied to an oxygen-deficient bismuth manganite structure with little a priori knowledge of the final structure (beyond the basic atomic positions of an ideal Ruddlesden-Popper structure). This metric can be applied across all samples without additional structural information (e.g. thickness) and it is hoped that this study can be a catalyst for electron crystallographers to develop new approaches to account for dynamical effects that are ever-present in their data.

One significant area for future work is to extend the approach to include full 3D diffraction data rather than simply solving individual projected crystal structures. This type of data is now readily available through, for example, the automated diffraction tomography approach pioneered by Kolb et.al. (2007) and the incorporation of precession with this technique means that the validity we have suggested in this work should still be present when considering 3-D refinement of the structure. 3-D data allows the full crystal structure to be investigated (rather than isolated projections) and it has been shown that having more complete data over a suitable range of spatial frequencies greatly enhances the ability to solve and refine crystal structures (CasIUCr macros version 2.1.4: 2010/12/07 
carano et. al. 2010).

Acknowledgements The authors would like to thank EPSRC for financial support through grant number EP/HO17712.

\section{References}

Barnard, J. S. Eggeman, A. S. Sharp, J. White, T. A. \& Midgley, P. A. (2010). Phil. Mag. 90, 4711-4730.

Burla, M. C. Caliandro, R. Camalli, M. Carrozzini, B. Cascarano, G. L. De Caro, L. Giacovazzo, C. Polidori, G. Siliqi, D. \& Spagna R. (2007). J. Appl. Cryst. 40, 609-613.

Cascarano, G. L. Giacovazzo, C. \& Carrozzini, B. (2010). Ultramicroscopy, 111, 56-61

Ciston, J. Deng, B. Marks, L. D. Own, C. S. \& Sinkler, W. (2008). Ultramicroscopy, 108, $512-522$.

Eggeman, A. S. White, T. A. \& Midgley, P. A. (2010). Ultramicroscopy 110, 771-777.

Eggeman, A. S. Sundaresan, A. Rao, C. N. R. \& Midgley, P. A. (2011). J. Mater. Chem. 21 (39), 15417-15421.

Elcombe, M. Kisi, E. H. Hawkins, K. D. White, T. J. Goodman, P. \& Matheson, S. (1991) Acta Cryst. B47, 305-314.

Frigo, M. \& Johnson, S. G. (2005). Proc. IEEE, 93 (2), 216231.

Giacovazzo, C. (1998). 'Direct phasing in crystallography: Fundamentals and applications' Oxford Science Publications.

Haider, M. Uhlemann, S. Schwan, E. Rose, H. Kabius, B. \& Urban, K. (1998) Nature, 392, 768-769.

Hovmöller, S. Zou, X. D. \& Weirich, T. E. (2002). Adv. Imag. Elect. Phys., 123, 257-289.

Jansen, J. Tang, D. Zanderberg, H. W. \& Schenk, H. (1998). Acta Cryst. A54, 91-101.

Klein, H. \& David, J. (2010). Acta Cryst. A67, 297-302.

Kolb, U. Gorelick, T. Kübel, C. Otten, M. T. \& Hubert, D. (2007). Ultramicroscopy, 107, 507-513

Krivanek, O. L. Dellby, N. \& Lupini, A. R. (1999). Ultramicroscopy, 78, 1-11.

Levenberg, K. (1944). Quart. Appl. Math. 2, 164168.

IUCr macros version 2.1.4: 2010/12/07 
Marquadt, D. (1962). SIAM J. Appl. Math. 11, 431-441.

Midgley, P. A. \& Saunders, M. (1996). Contemp. Phys. 37, 441-456.

Pyrz, W. D. Blom, D. A. Vogt, T. \& Buttrey, D. J. (2008), Angew. Chem. 120, 2830-2833.

Saxton, W. O. Pitt, T. J. \& Horner, M. (1979). Ultramicroscopy 4 343-354.

Sleight, M. (1997). 'The use of convergent beam electron diffraction as a method for structure determination' PhD Thesis, University of Bristol, UK.

Smolin Y. I. (1970). Soviet Physics - Crystallography, 15, 36-37.

Vincent, R. \& Midgley, P. A. (1994). Ultramicroscopy 53, 271-282.

Vincent, R. Bird, D. M. \& Midgley, P. A. (1984). Phil. Mag. A 50, 745-.

White, T. A. Eggeman, A. S. \& Midgley, P. A. (2010). Ultramicroscopy 110, 763-770.

IUCr macros version 2.1.4: 2010/12/07 


\begin{tabular}{|c|c|c||c|c|}
\cline { 2 - 5 } \multicolumn{1}{c|}{} & \multicolumn{2}{c||}{ Refined } & \multicolumn{2}{c|}{ Ideal } \\
\hline Element & $\mathrm{x}$ & $\mathrm{y}$ & $\mathrm{x}$ & $\mathrm{y}$ \\
\hline $\mathrm{Er}$ & 0.874 & 0.350 & 0.875 & 0.353 \\
\hline $\mathrm{Ge}$ & 0.900 & 0.153 & 0.899 & 0.152 \\
\hline $\mathrm{O} 1$ & 0.805 & 0.200 & 0.804 & 0.196 \\
\hline $\mathrm{O} 2$ & 0.960 & 0.075 & 0.960 & 0.076 \\
\hline $\mathrm{O} 3$ & 0.064 & 0.346 & 0.065 & 0.339 \\
\hline $\mathrm{O} 4$ & 0.668 & 0.163 & 0.677 & 0.162 \\
\hline
\end{tabular}

Table 1. Refined and ideal atomic co-ordinates for erbium pyrogermanate. 


\begin{tabular}{|c|c|c|c||c|c|c|c|c|}
\hline \multicolumn{4}{|c||}{ Model } & \multicolumn{5}{c|}{ Refined } \\
\hline Element & Wyckoff & $\mathrm{x}$ & $\mathrm{y}$ & Element & Wyckoff & $\mathrm{x}$ & $\mathrm{y}$ & $\langle$ error $\rangle$ \\
\hline Ca1 & 4a & 0.000 & 0.252 & Bi1 & $4 \mathrm{a}$ & 0.00 & 0.22 & 0.01 \\
\hline Ca2 & $8 \mathrm{~b}$ & 0.188 & 0.741 & Bi2 & $8 \mathrm{~b}$ & 0.18 & 0.74 & 0.01 \\
\hline Ti1 & 8b & 0.099 & 0.249 & Mn1 & $8 \mathrm{~b}$ & 0.10 & 0.24 & 0.01 \\
\hline O1 & 4a & 0.000 & 0.812 & O1 & $4 \mathrm{a}$ & 0.00 & 0.76 & 0.06 \\
\hline O2 & $8 \mathrm{~b}$ & 0.197 & 0.696 & O2 & $8 \mathrm{~b}$ & 0.21 & 0.78 & 0.07 \\
\hline O3 & 8b & 0.086 & 0.538 & O3 & $8 \mathrm{~b}$ & 0.10 & 0.50 & 0.07 \\
\hline O4 & 8b & 0.038 & 0.110 & O4 & $8 \mathrm{~b}$ & 0.09 & 0.05 & 0.06 \\
\hline
\end{tabular}

Table 2. Reported and refined atomic co-ordinates for erbium pyrogermanate. Average errors were calculated using the approach in Vincent et al. (1984) 


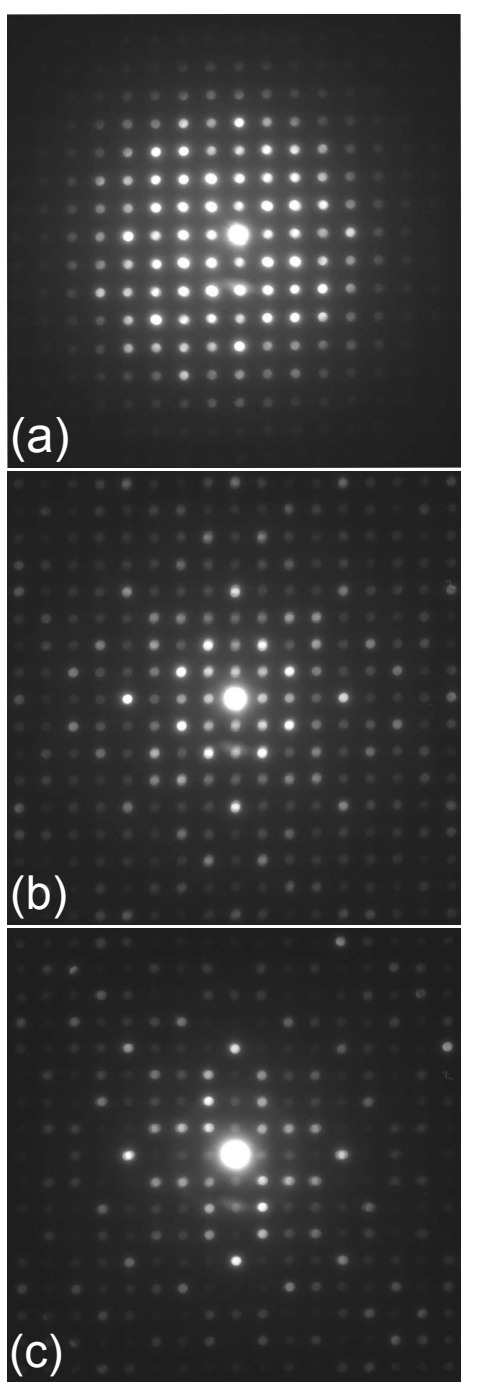

Fig. 1. Electron diffraction patterns from erbium pyrogermanate recorded parallel to [001] with a) 0, b) 20 and c) 50 mrad precession angle applied. 

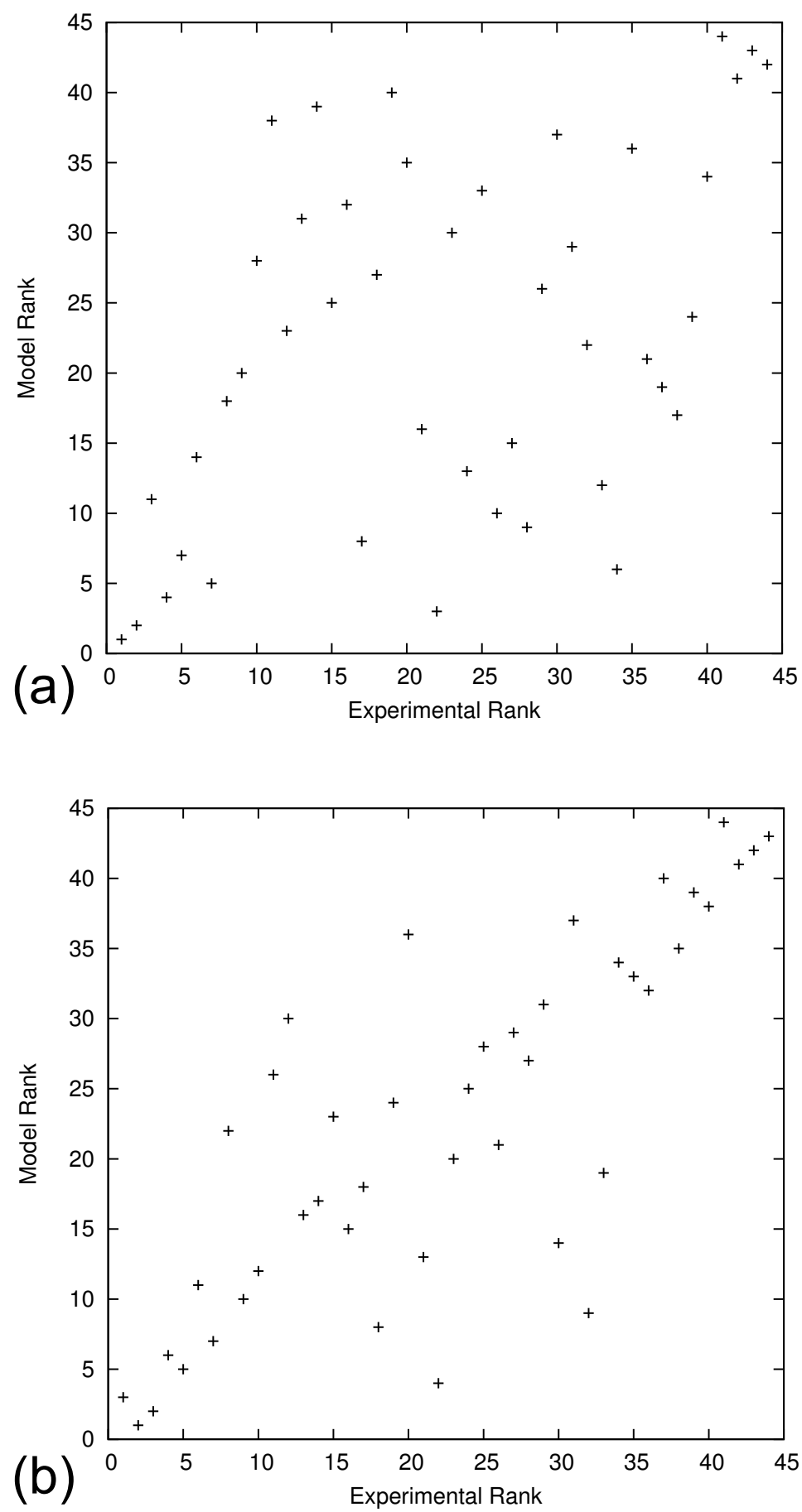

Fig. 2. Scatter-plots comparing the ranks of reflections generated from a) the initial and $b$ ) the refined structural model with the experimental reflection ranks for data recorded with $40 \mathrm{mrad}$ precession angle. The corresponding rank-correlation factors are 0.46 and 0.80 respectively. 


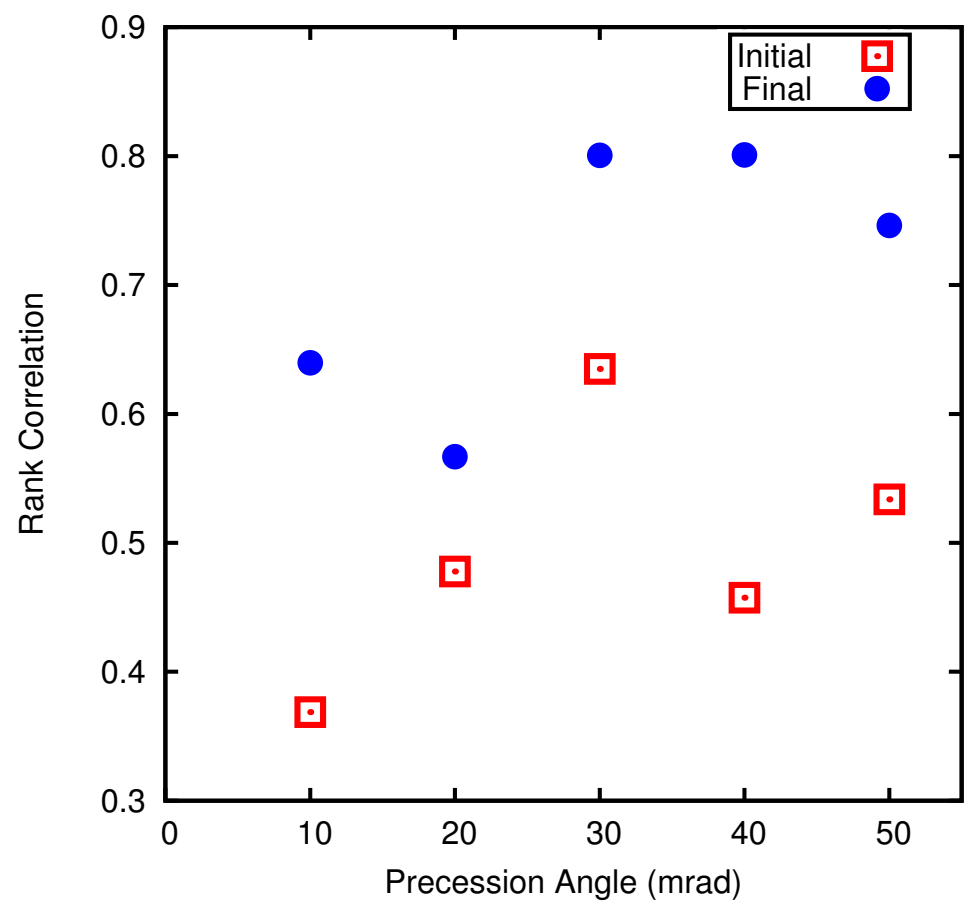

Fig. 3. Initial and final rank-correlation values for structural models as a function of precession angle. 

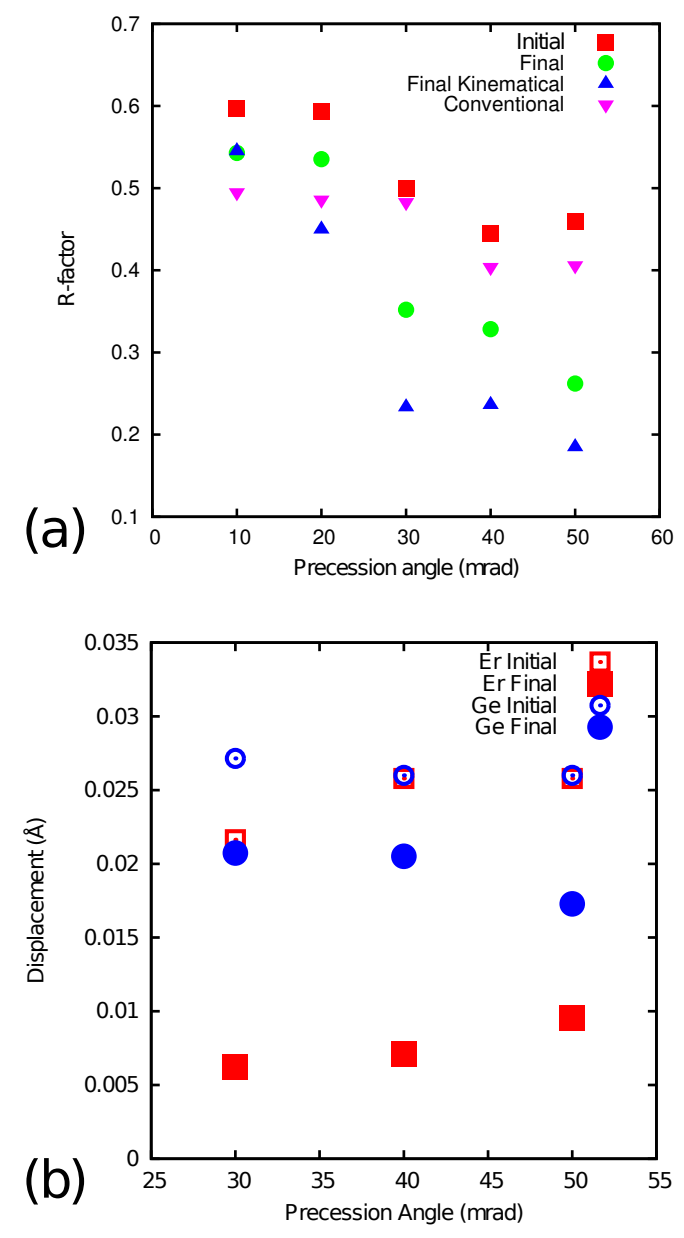

Fig. 4. a) Intensity residuals calculated for the initial structural models compared to the experimental diffraction intensities, for the refined structural models compared to the experimental diffraction intensities and for the refined models compared to the ideal kinematical diffraction intensities. Also shown are intensity residuals calculated for a conventional R-factor refinement of the structural model b) Atomic displacements from the ideal values for structural models as a function of precession angle before and after refinement using rank-correlation. 

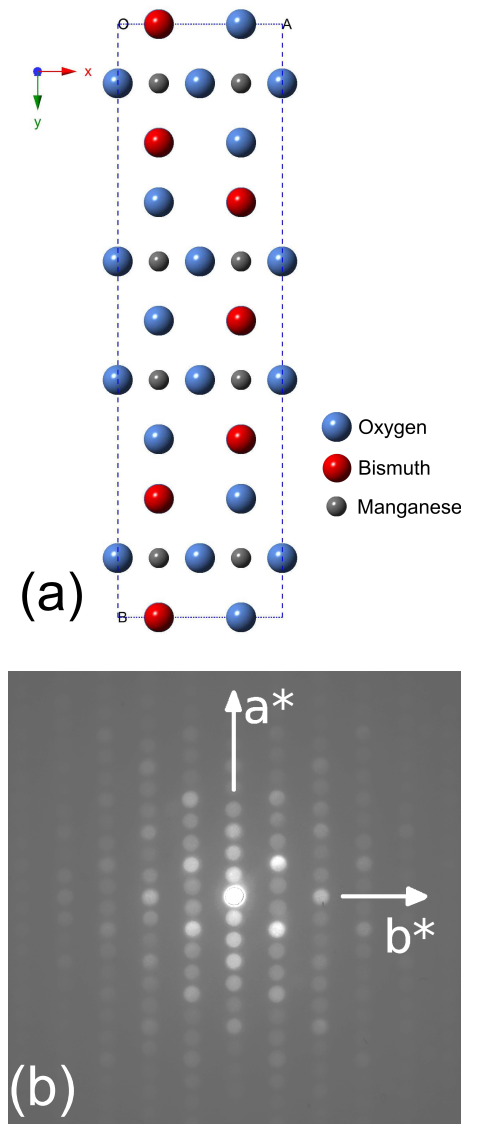

Fig. 5. a) Theoretical ideal model of an $n=2$ Ruddlesden-Popper structure. b) Electron diffraction pattern recorded parallel to [001] of a $\mathrm{BiMnO}_{2.91}$ crystal with $30 \mathrm{mrad}$ precession angle. 

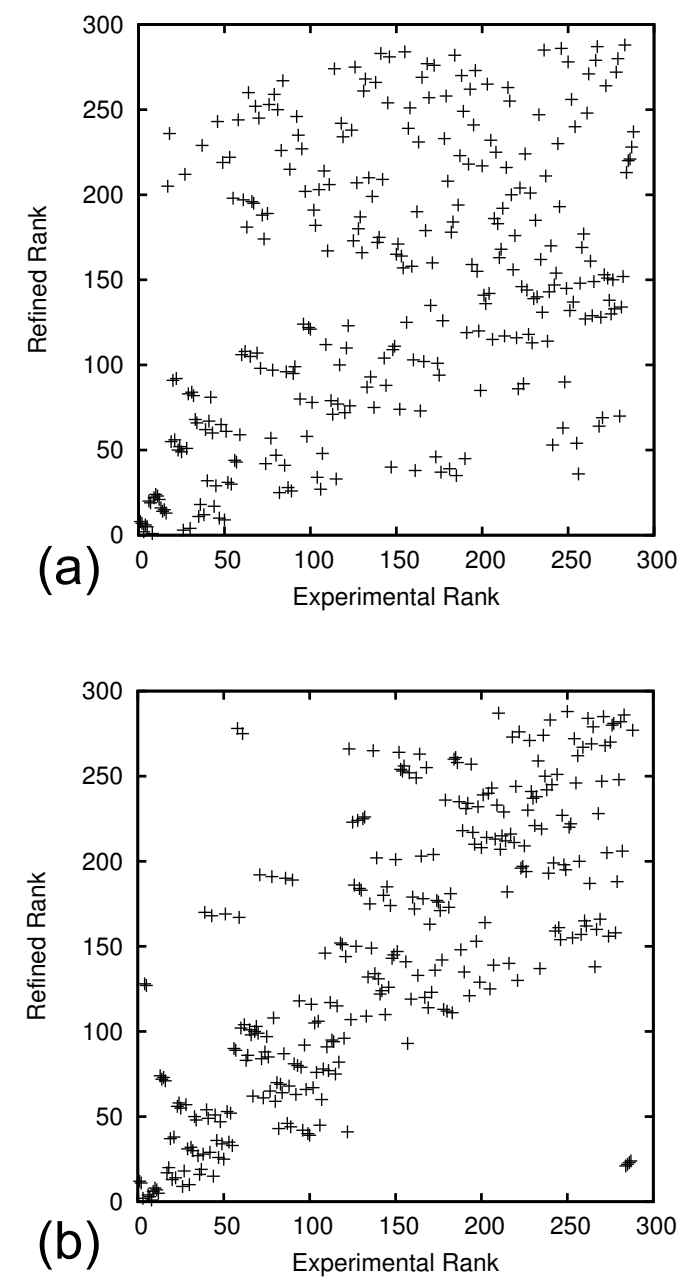

Fig. 6. Scatter plots of simulated reflection ranks against experimental reflection ranks for a) the initial bismuth manganite model and b) the refined bismuth manganite model. 


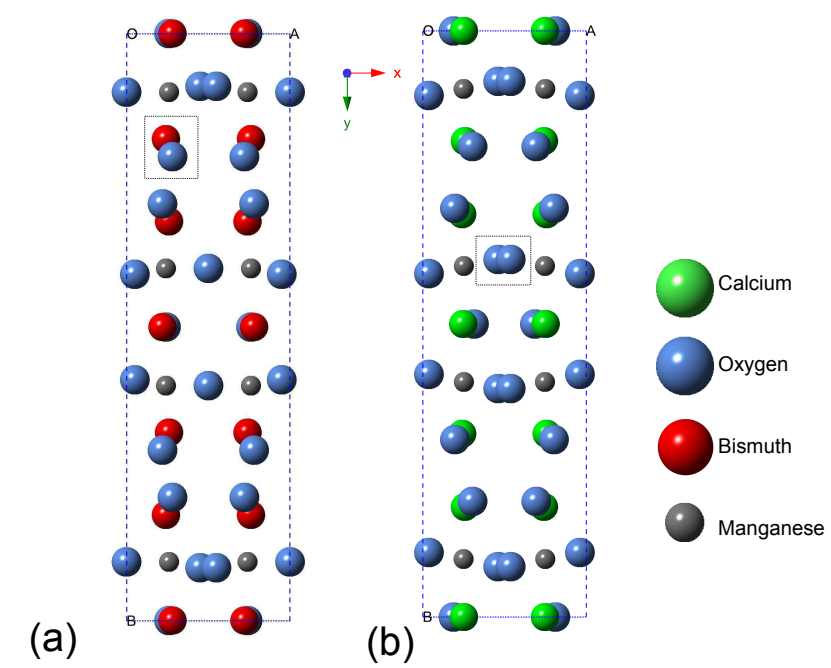

Fig. 7. a) Structure of $\mathrm{BiMnO}_{2.91}$ projected parallel to [001], refined against the electron diffraction pattern shown in Figure 5b. b) The same projection of the calcium manganite analogue structure.

\section{Synopsis}

The relative intensity (or rank) of a reflection is considered as an alternative metric when attempting structural refinement using precession electron diffraction data. 